\title{
Examination of preterm babies for ROP referred to an eye department from an urban and semi-urban neonatal intensive care unit: implications for practice.
}

\section{Ramesh Venkatesh ( $\square$ vramesh80@yahoo.com )}

Dr Shroff's Charity Eye Hospital https://orcid.org/0000-0002-4479-9390

\section{Prachi Abhishek Dave}

Dr Shroff's Charity Eye Hospital

\section{Prachi Gurav}

Dr Shroff's Charity Eye Hospital

\section{Manisha Agarwal}

Dr Shroff's Charity Eye Hospital

Mamta Jajoo

Chacha Nehru Bal Chikitsalaya

\section{Vidit Gupta}

B LAL Children Hospital

\section{Research article}

Keywords: prevalence, retinopathy of prematurity, rural, semi-urban, urban

Posted Date: September 18th, 2019

DOI: https://doi.org/10.21203/rs.2.9416/v3

License: (c) (i) This work is licensed under a Creative Commons Attribution 4.0 International License.

Read Full License 


\section{Abstract}

Background: To study and analyse the factors affecting the proportion of retinopathy of prematurity (ROP) distribution between the urban and semi-urban regions in North India. Methods: Retrospective, observational, cross-sectional study. All babies referred for ROP examination by paediatricians or other general ophthalmologists between 2013 - 2016 were included in the study. Demographic, clinical and treatment related findings were recorded. Results: Five hundred and fifty-eight (467: urban \& 91: semi urban) babies were examined for ROP. The mean birth weight in the urban and semi-urban setting was $1348.6 \pm 395.21 \mathrm{gm}$ and $1703.77 \pm 401.76 \mathrm{gm}$ respectively. The mean gestational age was $30.99 \pm 2.93$ weeks and $30.73 \pm 2.08$ weeks in the urban and semi-urban cohorts respectively. The average time for first ophthalmic examination following birth was $23.82 \pm 13.69$ (range: $3-77$ ) days in the urban and $101.16 \pm 238.26$ (range: $13-330$ ) days in the semi-urban setting. $94 \%$ of the babies completed all screening examination visits. Any ROP was identified in $11.6 \%$ and $33.0 \%$ of the urban and semi-urban cohorts respectively; Type 1 was detected in $7.5 \%$ of urban babies and $23.1 \%$ of semi-urban babies Conclusion: Differences in the proportion of babies developing any ROP and Type 1 ROP between the semi-urban and urban groups is likely due to selection bias, as a high proportion of semi-urban babies did not attend for examination or failed to complete all the examinations necessary. This was particularly true for females. More needs to be done to increase access to regular, systematic screening of preterm babies within neonatal units.

\section{Background}

Retinopathy of prematurity (ROP) is a retinal vascular condition, commonly seen in preterm, low birthweight babies. India leads all nations in the number of preterm infants who survive annually, making ROP a leading public health problem which has not been completely addressed. [1] The prevalence of ROP in India is reported at 24-47\%. [2-5] The profile of babies who develop ROP in the developing countries are older and heavier babies. [6,7] This is different in comparison to that of the developed countries; thereby increasing the burden of babies who need to be included for screening in our population. Until recently, most of the reports regarding the prevalence and profile of ROP babies in India have come from tertiary centres in bigger cities. However, prevalence of ROP reported recently from several rural and outreach centres from Karnataka state, indicate that ROP in these babies is as high if not higher than urban centres. [8-11] With variable level of neonatal care in some of these outreach zones, the severity of ROP, including severe zone 1 disease, is likely to be high. [9]

The recent Supreme Court of India judgement on the medicolegal negligence of the paediatrician on not providing for ROP screening on time, has enhanced the awareness with specialists and parent groups alike. [12] An acute shortage of qualified ROP specialists across the nation further worsens the situation. Hence, more babies need to be screened with limited resources.

In this study, we aim to compare the prevalence and profiles of ROP babies screened in two cities in North India and analyse the factors responsible for it. 


\section{Methods}

In this study, babies screened for ROP in 2 different states between August 2013 and September 2016 were included. Institutional review board (SCEH-2013-09-003) and ethics committee clearance was obtained for this study and informed written consent of the parents/child's guardian for participation in the study were also obtained. In our study, ROP screening was conducted in the retina clinic of a tertiary eye care institute on fixed days of the week - (on Monday's in Delhi and on Thursday's in Alwar). All the medical professionals, including the paediatricians and other ophthalmologists in the surrounding areas were informed regarding the screening guidelines via regular meetings and providing study materials. As per the National ROP guidelines, infants born $<1750 \mathrm{~g}$ at birth and/or $<34$ weeks of gestation were enrolled into the study. [13] Babies were referred to the retina clinic after the stabilisation of their systemic condition. For premature babies requiring long duration of NICU stay, screening was conducted in their respective neonatal care units. In our study, we primarily received referrals from 2 NICUs - urban NICU at the Chacha Nehru Bal Chikitsalaya (CNBC), New Delhi and semi-urban NICU at B. LAL Children Hospital, Alwar, Rajasthan. The NICU of CNBC at New Delhi is a level 3, out born tertiary care intensive care unit while that at Alwar was a level 2 out born NICU. Data recording at the initial examination included: 1 ) premature infant's gender; 2) time for first ophthalmic examination; 3) birth weight (BW) and gestation age (GA); 4) presence, stage and zone of ROP; 5) presence of plus disease and 6) treatment required if any. Eye examinations of all babies were done by a single examiner to standardize the examination technique and the results were analysed. The intent of the initial examination was to screen and detect babies with ROP and enrol them in the study. Ophthalmic examination was done with an indirect ophthalmoscope and a +20D lens at weekly intervals. Eyes were examined with an infant speculum and a wire vectis as scleral depressor, under topical anaesthesia using $2 \%$ proparacaine drops. The pupils were dilated by using $0.4 \%$ tropicamide and $2.5 \%$ phenylephrine eye drops two or three times, till full dilatation occurred. Retinopathy was graded into stages and zones as per the ICROP classification. $[14,15]$ Infants with normal vascularization up to the periphery were not examined again. Those with ROP were examined every week/2 weeks till regression occurred or till they reached threshold for laser treatment. Treatment in the study was performed by laser photoablation using the Early Treatment for Retinopathy of Prematurity guidelines with the 532-nm green laser. [16] As far as possible, the treatment was performed at the base hospital (Delhi) by the vitreo-retina specialist. Babies requiring laser treatment in the semi-urban set-up were shifted to the base hospital in Delhi on the same day or after discharge from NICU with clearance from the treating paediatrician. For babies in the urban set-up requiring treatment, laser therapy was carried out on the same or next day at our base hospital. Once the babies were discharged from the NICU, they were followed up in the out-patient clinic of the retina department for further evaluation. If regression was found to be inadequate or skip areas were seen on subsequent examination, laser was repeated after one or two weeks. Intravitreal Bevacizumab injection was given to babies diagnosed with aggressive posterior ROP.

\section{Statistical Analysis:}


Analysis was performed using SPSS version 22.0 for Macbook. Univariate analysis was conducted using Chi square test. Multiple logistic regression analysis was performed to study the predictors of ROP using independent variables as those variables which were significant in the univariate analysis.

\section{Results}

During the period from August 2013 and September 2016, a total of 721 (240 - semi-urban; 481 - urban) preterm babies satisfied the inclusion criteria for screening of which 558 babies were screened for ROP. Out of the 558 babies screened, 467 babies belonged to the urban cohort and 91 babies to the semi-urban cohort. More males underwent screening for ROP than girls (376 males and 182 females) in both urban and semi-urban settings. The mean BW in the urban and semi-urban setting was $1348.6 \pm 395.21 \mathrm{gm}$ and $1703.77 \pm 401.76 \mathrm{gm}$ respectively. The mean GA of the premature babies was $30.99 \pm 2.93$ weeks and $30.73 \pm 2.08$ weeks in the urban and semi-urban cohorts respectively. The average time for first ophthalmic examination following birth was $23.82 \pm 13.69$ days in the urban and $101.16 \pm 238.26$ days in the semi-urban setting. Overall prevalence of ROP was $15.1 \%$ (84) and it was not significantly influenced by gender $(p=0.514)$. Prevalence of ROP in the urban cohort was $11.7 \%(55)$ and in the semiurban cohort was $31.9 \%$ (29). There was statistically significant difference between the two groups with respect to birth weight, day of screening and prevalence of ROP but not statistically significant to gender or GA (table 1). Stages of ROP identified in the urban and semi-urban groups is described in table 2 . Four babies in the semi-urban cohort and 13 babies in the urban cohort were identified with plus disease. Aggressive posterior ROP (APROP) was noted in 1 preterm baby in the semi-urban group and 5 babies in the urban group. In the urban group, severe ROP was identified in 35 babies while 21 babies in the semiurban group had severe treatable ROP. $85.7 \%$ babies in the urban and semi-urban cohorts underwent treatment for different stages of ROP. Out of 84 babies who developed ROP, 41 babies required laser alone and 6 babies required laser and anti-vascular endothelial growth factor (VEGF) therapy; 30 (55.5\%) belonged to the urban cohort and $17(58.6 \%)$ to the semi-urban cohort. There was statistically significant difference in the prevalence of severe ROP between the urban and semi-urban cohorts $(p=0.000)$. Using the multivariate regression model, independent risk factors like GA, birthweight, day of screening, semiurban or urban setting and gender were correlated with the presence or absence of ROP (table 3 ).

\section{Discussion}

In our study, the overall prevalence of ROP was $15.1 \%$. We found a statistically significant higher prevalence of ROP in babies with $\mathrm{BW}<1500 \mathrm{~g}, \mathrm{GA}<28$ weeks, screening before 1 month of age and in the semi-urban setting. The prevalence of ROP was almost 3 -fold higher in the semi-urban setting (31.9\%) compared to the urban setting $(11.7 \%)$. Also, the drop-out rate for ROP screening was higher in the semiurban setting. In this study, we analysed the profile of ROP babies and also explain the possible reasons for this unequal distribution of ROP in the 2 settings.

In our study, more boys ( $n=376)$ underwent screening for ROP compared to girls $(n=182)$. This trend was seen both in the urban and semi-urban cohorts. One of the more probable reasons could be that the 
parents consider the boys to be more valuable and hence, they bring them for screening. In the semiurban setting, the median time interval between birth and first day of screening of these premature babies was 60 (mean $=101)$ days while in the urban setting it was 21 (mean $=24)$ days. Jalali et al [17] had discussed the role of early screening strategy for preterm babies. Their study suggested early screening before one month of age in neonatal centres can detect early disease and prompt treatment can lead to favourable outcomes. In our study, we found the mean GA to be similar in both groups while in semiurban setup more heavier babies were screened for ROP $(1348.6 \pm 395.21 \mathrm{gm}$ in urban; $1703.77 \pm 401.76$ $\mathrm{gm}$ in semi-urban setting). Our study revealed higher prevalence of stage 5 ROP with total inoperable retinal detachment in 7 eyes in the semi-urban cohort and only 2 eyes in the urban population; thereby further stressing on the need for early screening for ROP to detect the disease early.

Only 91 babies of the eligible 240 babies underwent screening for ROP in the semi-urban cohort accounting to a drop-out rate of about $62 \%$ while the drop-out rate in the urban cohort was only $2.9 \%$. In India, currently, most of the ROP screenings are done by retina specialists. Paediatric ophthalmology is still not a well-established separate subspecialty in India. $[18,19]$ Most of the vitreo-retinal surgeons and paediatric ophthalmologists practise in bigger cities. As a result, there is a paucity of ROP care givers in these areas. Also, the profile of babies who develop ROP in developing countries like ours are older and heavier babies. $[6,7]$ This further increases the burden of babies who need to be included for screening in our population. Hence, preterm babies born in the semi-urban/rural areas are not being subjected for ROP screening as per the screening guidelines. Studies have shown paediatricians and ophthalmologists practising in the interiors of the country to have poor knowledge regarding ROP and its screening strategies. $[20,21]$ Creating awareness amongst the medical staff like ophthalmologists, paediatricians, gynaecologists and nurses in the NICU and providing training and basic infrastructure like indirect ophthalmoscopy for screening preterm babies can increase the overall screening rate. Also stressing the need for screening at the time discharge to parents/guardians can help in increasing the ROP screening rate in rural/semi-urban areas. Lack of availability of treatment facilities like laser in these areas could be responsible for the high drop-out rate as well. Providing treatment to such high-risk babies at the screening site would be beneficial in preventing childhood blindness due to ROP. In our study, we did screen preterm babies from 2 population groups who were socially and economically unequal.

The quality of neonatal care provided in the urban and semi-urban NICUs is highly variable. The urban NICU at Delhi is a level IIIA NICU providing a high level of neonatal care to very low birth-weight premature babies. They have a wide variety of trained staff available on site including neonatologists, neonatal nurses and respiratory therapists who are available 24-hours a day. In comparison, the semi-urban NICU at Alwar is a level II NICU providing care to infants who are moderately ill with problems that are expected to resolve rapidly. [22] Care in this setting is usually limited to new-born infants who are $>32$ weeks' GA and weigh $>1500 \mathrm{~g}$ at birth or who are recovering from serious illness treated in a level III NICU. Chow et al [23] had observed a significant decrease in the prevalence of ROP with training of the NICU staff and better implementation and enforcement of clinical practices related to oxygen (02) management and monitoring. In their study, the prevalence of ROP decreased consistently in a 5 -year period from $12.5 \%$ in 1997 to $2.5 \%$ in 2001 . The need for ROP laser treatment decreased from $4.5 \%$ in 1997 to $0 \%$ over the last 
3 years. Even in our study we found a significantly lower prevalence of ROP in the urban setting due to the high level of neonatal care provided, increased awareness for early screening of preterm babies and better understanding of the disease amongst the paediatricians.

Our study highlights important key issues related to ROP screening and treatment in the urban and semiurban population settings. This study stresses the need for more and more medical personnel, especially those belonging to the semi-urban/rural setups to be trained for ROP screening by premier tertiary eye care institutions. This, in turn will reduce the ROP burden and childhood blindness in our country.

\section{Conclusion}

The prevalence of ROP was found to be higher in the semi-urban setting due to delayed screening, higher drop-out rate and lack of basic treatment facilities. Stricter adherence to the screening protocols and creating awareness amongst paediatricians and parents regarding ROP can increase the screening rate in the semi-urban areas. Providing training to more medical professionals like paediatricians, general and pediatric ophthalmologists for ROP screening can reduce the drop-out rate. Also, better understanding of $\mathrm{O} 2$ management and monitoring in NICUs and providing basic treatment like laser at the NICU itself would reduce the prevalence of blindness due to ROP.

\section{Abbreviations}

1. ROP - retinopathy of prematurity

2. NICU - neonatal intensive care unit

3. BW - birth weight

4. GA - gestational age

5. APROP - aggressive posterior retinopathy of prematurity

6. VEGF - vascular endothelial growth factor

\section{Declarations}

1) Ethics approval and consent to participate - Approval has been obtained from the institutional review board (SCEH-2013-09-003) of Dr. Shroff's Charity Eye Hospital and ethics committee.

2) Consent for publication - written consent has been obtained from the parent/legal guardian during screening for $R O P$

3) Availability of data and materials - The datasets used and/or analysed during the current study are available from the corresponding author on reasonable request.

4) Competing Interests - The authors declare that they have no competing interests 


\section{5) Funding - None}

\section{6) Authors' Contribution -}

$R V$ - conducting the screening, analysing the results, writing the manuscript and reviewing the paper. PDJ and $P G$ - data entry and screening of babies. MA, MJ and VG - reviewing the paper. All authors have read and approved the manuscript.

\section{7) Acknowledgements - Not Applicable References}

1) Varughese S, Jain S, Gupta N, Singh S, Tyagi V, Puliyel JM. Magnitude of the problem of retinopathy of prematurity. experience in a large maternity unit with a medium size level-3 nursery. Indian $\mathrm{J}$ Ophthalmol. 2001 Sep;49(3):187-8.

2) Charan R, Dogra MR, Gupta A, Narang A. The Prevalence of retinopathy of prematurity in a neonatal care unit. Indian J Ophthalmol 1995; 43:123-26.

3) Gopal L, Sharma T, Ramachandran S, Shanmugasundaram R, Asha V. Retinopathy of prematurity: a study. Indian J Ophthalmol 1995; 43:59-61.

4) Rekha S, Battu RR. Retinopathy of prematurity: prevalence and risk factors. Indian Paediatrics 1996; 33:999-1003.

5) Varughese S, Jain S, Gupta N, Singh S, Tyagi V, Puliyel JM. Magnitude of the problem of retinopathy of prematurity. Experience in a large maternity unit with a medium size level 3 nursery. Indian $\mathrm{J}$ Ophthalmol $2001 ; 49: 187$.

6) Vinekar A, Dogra MR, Sangtam T, Narang A, Gupta A. Retinopathy of prematurity in Asian Indian babies weighing greater than 1250 grams at birth: ten-year data from a tertiary care centre in a developing country. Indian J Ophthalmol. 2007 Sep-0ct;55(5):331-6.

7) Jalali S, Matalia J, Hussain A, Anand R. Modification of screening criteria for retinopathy of prematurity in India and other middle-income countries. Am J Ophthalmol 2006; 141:966-68.

8) Vinekar A, Jayadev C, Mangalesh S, Shetty B, Vidyasagar D. Role of tele-medicine in retinopathy of prematurity screening in rural outreach centres in India - a report of 20,214 imaging sessions in the KIDROP program. Semin Fetal Neonatal Med. 2015 Oct;20(5):335-45.

9) Vinekar A, Jayadev C, Dogra M, Shetty B. Improving Follow-up of Infants during Retinopathy of Prematurity Screening in Rural Areas. Indian Pediatr. 2016 Nov 7;53 Suppl 2:S151-S154.

10) Hungi B, Vinekar A, Datti N, Kariyappa P, Braganza S, Chinnaiah $S$ et al. Retinopathy of prematurity in a rural Neonatal Intensive Care Unit in South India-a prospective study. Indian J Pediatr 2012; 79:911-15. 
11) Murthy KR, Murthy PR, Shah DA, Nandan MR, S NH, Benakappa N. Comparison of profile of retinopathy of prematurity in semiurban/rural and urban NICUs in Karnataka, India. Br J Ophthalmol 2013;97:687-689.

12) Available from: http://www.supremecourtofindia.nic.in/jonew/judis/42745.pdf Accessed on 21st July 2017.

13) Pejaver RK, Vinekar A, Bilagi A [webpage on the Internet]. National Neonatology Foundation's Evidence Based Clinical Practice Guidelines 2010. Retinopathy of Prematurity (NNF India, Guidelines); 2010: 253-262. Available from: http://aimaonline.org/iap-neochap-2013/uploads/ acdcorner/nnf_guidelines-2011.pdf. Accessed on 21st July 2017.

14) International Committee for the Classification of Retinopathy of Prematurity. Multicentre trial of cryotherapy. An international classification of retinopathy of prematurity. Arch Ophthalmol 1984; 102: 1130-1134.

15) International Committee for the Classification of the Late Stages of Retinopathy of Prematurity. An international classification of retinopathy of prematurity II. The classification of retinal detachment. Arch Ophthalmol 1987; 105: 906-912.

16) Early Treatment for Retinopathy of Prematurity Cooperative Group. Revised indications for the treatment of retinopathy of prematurity: results of the early treatment for retinopathy of prematurity randomized trial. Arch Ophthalmol. 2003;121(12):1684-1694.

17) Subhadra Jalali, Raj Anand, Padmaja Kumari Rani, Divya Balakrishnan. Impact of the day-30 screening strategy on the disease presentation and outcome of retinopathy of prematurity. The Indian twin cities retinopathy of prematurity report number 3. Indian J Ophthalmol. 2014 May;62(5):610-4.

18) Nirmalan PK, Sheeladevi S, Tamilselvi V, Victor AC, Vijayalakshmi P, Rahmatullah L. Perceptions of eye diseases and eye care needs among parents in rural South India: the Kariapatti Pediatric Eye Evaluation Project (KPEEP). Indian J Ophthalmol 2004; 52:163-7.

19) Vijaylakshmi P, Nirmalan P, Kothari MT. Pediatric ophthalmology and strabismus in India. J AAPOS 2004; 8:18-19.

20) Patwardhan SD, Azad R, Gogia V, Chandra P, Gupta S. Prevailing clinical practices regarding screening for retinopathy of prematurity among paediatricians in India: a pilot survey. Indian J Ophthalmol. 2011 Nov-Dec;59(6):427-30.

21) Sathiamohanraj SR, Shah PK, Senthilkumar D, Narendran V, Kalpana N. Awareness of retinopathy of prematurity among paediatricians in a tier two city of South India. Oman J Ophthalmol. 2011 May;4(2):77-80.

22) Bhatt D. Towards a unified definition of NICU levels of care. Perinat Sect News.2001;7:12 
23) Chow LC, Wright KW, Sola A. CSMC Oxygen Administration Study Group. Can changes in clinical practice decrease the prevalence of severe retinopathy of prematurity in very low birth weight infants? Paediatrics. 2003;111(2):339-45.

\section{Tables}

Table 1: Descriptive Data of babies screened for ROP in Urban and Semi-urban setting

\begin{tabular}{llll} 
& Urban & Semi urban & P value \\
\hline Eligible babies for screening & 481 & 240 & \\
\hline Babies who underwent screening & 467 & 91 & \\
\hline Gender & & & 0.514 \\
\hline Male & 315 & 61 & \\
\hline Female & 152 & 30 & 0.000 \\
\hline Birth weight (gm) & $1348.6 \pm 395.21$ & $1703.77 \pm 401.76$ & 0.512 \\
\hline Gestational age (weeks) & $30.99 \pm 2.93$ & $30.73 \pm 2.08$ & 0.000 \\
\hline First Ophthalmic examination (Days) & $23.82 \pm 13.69$ & $101.16 \pm 238.26$ & \\
\hline ROP present & & & 0.000
\end{tabular}

Table 2: Profile of ROP Babies and treatment given in urban and semi-urban cohorts: 


\section{Urban ( $\mathrm{N}=$ no. of babies $) \quad$ Semi-urban $(\mathrm{N}=$ no. of babies $)$}

\section{ROP Stages}

\begin{tabular}{lll} 
Stage 1 & 19 & 2 \\
\hline Stage 2 & 6 & 7 \\
\hline Stage 3 & 28 & 15 \\
\hline Stage 4 & 1 & 4 \\
\hline Stage 5 & 1 & 4 \\
\hline Plus disease & 13 & \\
\hline Treatment offered to & 35 & 21 \\
\hline Treatment taken by & 30 & 18 \\
\hline Treatment options & 33 & 8 \\
\hline Observe & 25 & 16 \\
\hline Laser & 5 & 1 \\
\hline Laser + Anti-VEGF & 0 & 1 \\
\hline Surgery & 0 & 5 \\
\hline ROP babies with poor outcome & 30 & 17 \\
\hline ROP babies with laser with good & & \\
\hline Outcome & & \\
\hline
\end{tabular}

Table 3: Correlating the independent risk factors with the presence or absence of ROP using the multivariate regression model: 
ROP ROP P value

Present Absent

(n) (n)

\begin{tabular}{lccc}
\hline BW $<1500 \mathrm{gm}$ & 61 & 296 & 0.046 \\
\hline $\mathrm{BW}>1500 \mathrm{gm}$ & 23 & 178 & \\
\hline $\mathrm{GA}<28$ weeks & 43 & 100 & 0.000 \\
\hline $\mathrm{GA}>28$ weeks & 41 & 374 & \\
\hline Screening $<30$ days & 56 & 125 & 0.000 \\
\hline Screening $>30$ days & 28 & 349 & \\
\hline Urban setting & 54 & 513 & 0.000 \\
\hline Semi-urban setting & 30 & 61 & \\
\hline Male & & & \\
\hline Female & 54 & 322 & 0.295 \\
\hline
\end{tabular}

